\title{
Surface Plasmon Resonance of Nanoshell Particles with PMMA-Graphene Core
}

\author{
R. Tarparelli, R. Iovine, L. La Spada and L. Vegni \\ Department of Engineering, "Roma Tre" University, \\ Via della Vasca Navale 84, Rome 00146, Italy \\ richard.tarparelli@uniroma3.it
}

\begin{abstract}
In this contribution we propose a numerical investigation of a new type of nanoshell particles. The structure consists of a core/shell particle with a PMMA (PolyMethyl MethAcrylate)-Graphene core and Au thin shell. The proposed particles exhibit a strong near electric field intensification when an electromagnetic plane wave in the Visible regime is applied. The optical properties are evaluated and discussed in terms of resonant behavior and the near electric field distribution. Finally, the sensitivity performance of the structure is analyzed for biosensing applications.
\end{abstract}

Keywords-nanoparticles; nanoshell, PMMA - Graphene, biosensing.

\section{INTRODUCTION}

It is well known that metal nanoparticles are particularly suitable for various sensing approaches [1]. These applications arise from the interactions between electromagnetic field in the VIS (Visible) regime and the free electrons of metal nanostructures. When the electromagnetic field is applied an electron fluctuations is obtained. The resonant condition highly depends on refractive index of the background environment, the type of the metal and the geometry of the particle [2]. This phenomenon is called LSPR (Localized Surface Plasmon Resonance) due to the strong local electromagnetic interactions in a small area near the nanoparticle surface.

Recently, many studies have focused their attention on graphene for its unusual electrical, thermal, mechanical and optical properties. The influence of the electron graphene mobility depends on the substrate material on which it is transferred [3]. For example, the possibility to transfer graphene on the PMMA substrate is now well established and evaluated [4]. This technique allows to obtain a significant improvement in the electrical and mechanical properties (conductivity, thermal and mechanical stability) of the classical PMMA substrates [5].

In this work we propose for the first time a numerical investigation of a new type of nanoshell-particles based on PMMA-Graphene. These particles are modeled in our simulation with a PMMA-Graphene Core coated by a thin gold shell. The influence of the ratio between core and shell size on the optical properties are analyzed and discussed. In particular the evolution of the extinction cross-section spectra and the near electric field distribution for different values are obtained.

Finally, a sensitivity analysis by varying the refractive index background is conducted. The results clearly show good values for different geometrical configurations that indicate the possibility to use such particles for ultrasensitive biosensing applications.

\section{NUMERICAL ANALYSIS NANOSHELl PARTICLES}

The proposed particles consist of a core shell structure with PMMA-Graphene core and Au thin shell (Fig. 1). To estimate the optical properties of such a structure the finite integration commercial code CST Studio Suite has been used. The permittivity values for the PMMA-Graphene core are taken from the literature [6]. These values are related to the Drude optical conductivity of graphene transferred on PMMA substrate using spin-coating method. For gold shell the experimental values of complex permittivity dispersion as reported in [7] have been used. The surrounding dielectric medium is considered to be vacuum.

The structure is illuminated by an electromagnetic impinging plane wave as depicted in Fig. 1.
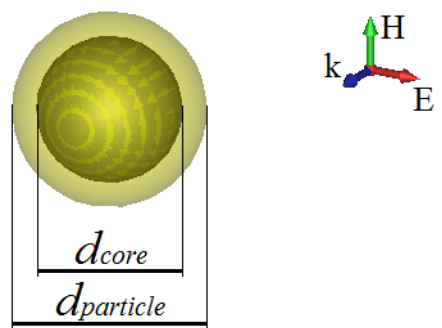

Fig. 1. Nanoshell particle with PMMA-Graphene Core and Au shell.

The electromagnetic response in terms of extinction cross-section and near electric field distribution is evaluated by varying geometrical parameters such as the diameter of the particle $\left(d_{\text {particle }}\right)$ and PMMA-Graphene core $\left(d_{\text {core }}\right)$.

As an example in Fig. 2 the dependence of the nanoparticle plasmon resonance wavelength on the nanoparticle size is shown.

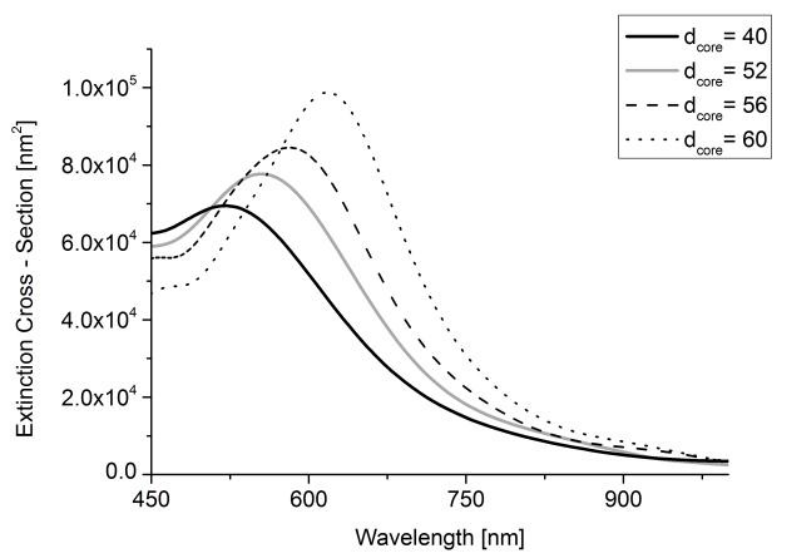

Fig. 2. Nanoshell extinction spectra for different value of the core size. The diameter of the particle is 70 . All dimensions are expressed in $\mathrm{nm}$. 
At the resonant wavelength the near electric field distribution (Fig. 3) confirms that the electromagnetic interaction between the proposed particle and the incident plane wave is restricted in a small area. This means that the local changes in the refractive index environment can be detected by the shift of the resonant wavelength of the structure. It is possible to observe (Fig. 3) that the electric field inside the core particle is very low due to the ENZ (Epsilon Near Zero) permittivity values at the resonant frequency of the PMMA-Graphene material.

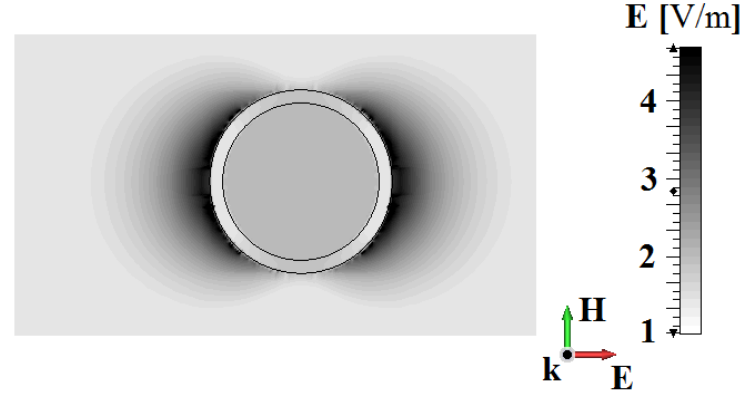

Fig. 3. Near electric field distribution of the core/shell particles at the resonant wavelength $(627 \mathrm{~nm})$ for $d_{\text {particle }}=70 \mathrm{~nm} ; d_{\text {core }}=60 \mathrm{~nm}$. The incident electric field amplitude is $1 \mathrm{~V} / \mathrm{m}$.

\section{SENSITIVITY ANALYSIS}

In this section we analyze the nanoparticle sensitivity properties by varying the geometrical parameters of the core/shell structure.

The sensitivity is commonly defined as the ratio $S=\Delta \lambda / \Delta n$ expressed in $\mathrm{nm} / \mathrm{RIU}$ (Refractive Index Unit). A test material, surrounding the nanoparticle, with a varying refractive index $n$ in the range 1-1.6 has been chosen.

In Fig. 4 an example of the extinction spectra evolution for different values of the RI (Refractive Index) environment is depicted.

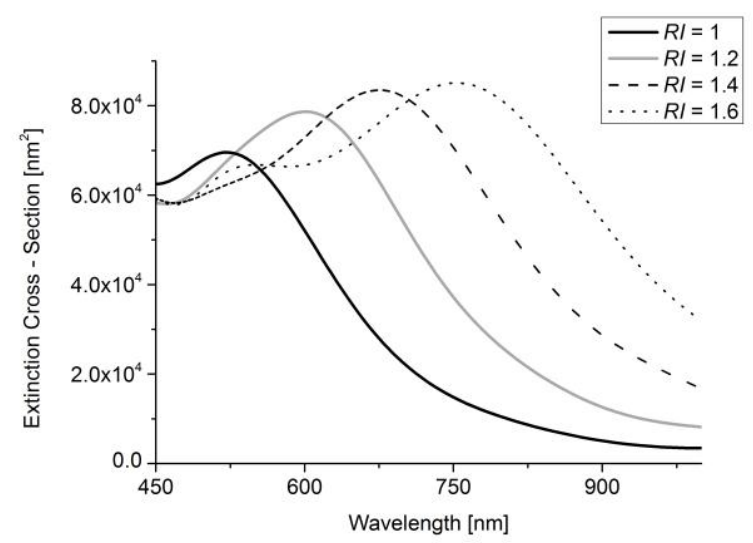

Fig. 4. Nanoshell extinction spectra for different value of the Refractive Index $(R I)$ background environment.

The particles size are: $d_{\text {particle }}=70 \mathrm{~nm} ; d_{\text {core }}=40 \mathrm{~nm}$.

To evaluate the sensitivity performance for different core/shell sizes extensive simulations have been performed. In TABLE 1 the mean sensitivity values for different configuration are clearly shown.
TABLE I. Sensitivity performance of nanoshell particles for different values of the core/shell sizes.

\begin{tabular}{|c|c|c|c|c|c|}
\hline \multirow{2}{*}{$\begin{array}{c}\text { Particle size } \\
{[\mathrm{nm}]}\end{array}$} & \multicolumn{4}{|c|}{$\begin{array}{c}\text { Resonant wavelength } \\
\lambda[\mathrm{nm}]\end{array}$} & $\begin{array}{c}\text { Mean } \\
\text { Sensitivity } \\
\text { Values } \\
{[n+1.2}\end{array}$ \\
\cline { 2 - 6 } & $n=1$ & $n=1.2$ & $n=1.4$ & $n=1.6$ & 390 \\
\hline $\begin{array}{c}d_{\text {particle }}=70 \\
d_{\text {core }}=40\end{array}$ & 521 & 602 & 673 & 755 & 388 \\
\hline $\begin{array}{c}d_{\text {particle }}=70 \\
d_{\text {core }}=44\end{array}$ & 529 & 606 & 685 & 762 & 388 \\
\hline $\begin{array}{c}d_{\text {particle }}=70 \\
d_{\text {core }}=48\end{array}$ & 540 & 622 & 691 & 770 & 375 \\
\hline $\begin{array}{c}d_{\text {particle }}=70 \\
d_{\text {core }}=52\end{array}$ & 556 & 638 & 711 & 787 & 385 \\
\hline $\begin{array}{c}d_{\text {particle }}=70 \\
d_{\text {core }}=56\end{array}$ & 583 & 661 & 740 & 822 & 398 \\
\hline $\begin{array}{c}d_{\text {particle }}=70 \\
d_{\text {core }}=60\end{array}$ & 617 & 698 & 778 & 870 & 421 \\
\hline
\end{tabular}

The numerical results of TABLE I indicate a mean sensitivity values of $400 \mathrm{~nm} / \mathrm{RIU}$. This good value encourages the possibility to use such a structure for biosensing applications.

\section{CONCLUSION}

In this contribution a computational investigation of PMMA-Graphene/Au nanoparticles operating in the VIS regime was proposed.

The optical behavior of the nanoshell particles are evaluated through full-wave numerical analysis. In particular, the extinction cross section spectra, the near electric field distribution and sensitivity performance were presented.

The preliminary results indicate that such particles could be used as an useful tool for ultra-sensitive biosensing applications.

\section{REFERENCES}

[1] K. Saha, S.S. Agasti, C. Kim, X. Li, and V.M. Rotello, "Gold Nanoparticles in Chemical and Biological Sensing," Chemical Reviews, vol. 112, pp. 2739-2779, 2012.

[2] P.K. Jain, K.S. Lee, I.H. El-Sayed, and M.A. El-Sayed, "Calculated Absorption and Scattering Properties of Gold Nanoparticles of Different Size, Shape, and Composition: Applications in Biological Imaging and Biomedicine," J. Phys.: Chem B, vol. 110, pp. 72387248, 2006.

[3] K.S. Kim, Y. Zhao, H. Jang, S.Y. Lee, J.M. Kim, K.S. Kim, J.H. Ahn, P. Kim, J.Y. Choi, and B.H. Hong, "Large-scale pattern growth of graphene films for stretchable transparent electrodes," Nature, vol. 457, pp. 706-710, 2009.

[4] V.H. Pham, T.T. Dang, S.H. Hur, E.J. Kim, and J.S. Chung, "Highly Conductive Poly(methyl methacrylate) (PMMA)-Reduced Graphene Oxide Composite Prepared by Self-Assembly of PMMA Latex and Graphene Oxide through Electrostatic Interaction," App. Mat. \& Int., vol.4, pp. 2630-2636, 2012.

[5] J. Wang, H. Hu, X. Wang, C. Xu, M. Zhang, and X. Shang, "Preparation and Mechanical and Electrical Properties of Graphene Nanosheets-Poly(methyl methacrylate) Nanocomposites via In Situ Suspension Polymerization," J. Appl. Polym. Sci., vol. 122, pp.18661871, 2011

[6] J. Y. Kim, J. H. Rho, C. Lee, S. Bae, S. J. Kim, K. S. Kim, B. H. Hong, and E. J. Choi, "Infrared conductivity and carrier mobility of large scale graphene on various substrates," J. Nanosci. Nanotech. vol.12, pp. 5816-5819, 2012.

[7] P.B. Johnson, and R.W. Christy, "Optical Constants of the Noble Metals," Phys. Rev. B, vol.6, pp. 4370-4379, 1972. 